Baltic Astronomy, vol.4, 482-496, 1995.

\title{
THE WET STANDARD PHOTOMETER
}

\author{
S. J. Kleinman, R. E. Nather and T. Phillips
}

Astronomy Department, University of Texas, Austin TX 78712, U.S.A.

Received September 1, 1995.

\begin{abstract}
Uniformity of instrumentation is a vital requirement for the success of the WET network. We report here on the photometer adopted in the previous WET conference as the standard WET photometer. In particular, we describe a recent addition to the design which allows the previously two-channel instruments to be converted to three-channel instruments, the third channel constantly measuring the sky background. This new component is a bolt-on upgrade to the existing two-channel photometers used by the WET and many others around the world. We will make the complete design (including acquisition and reduction software) available to interested parties upon request
\end{abstract}

Key words: instrumentation: photometers - stars: interiors - stars: oscillations - stars: individual: PG Vir, PG 1159, G 29-38, GD 358.

\section{Introduction}

In the beginning of the modern era of time-series photometry, there was the one-channel photometer adopted unchanged from the traditional $U B V$ photometrists: a single phototube continuously measuring the brightness of a single star. Unfortunately, the single detector made it difficult, or impossible, to distinguish true variability from such local effects as clouds. Thus, the two-channel photometer was developed (Nather 1973, Grauer \& Bond 1981) to measure two stars simultaneously. If the second star was chosen to be a nonvariable, then any variability that showed up in both channels was an artifact and not intrinsic to the observed stars.

Initially, these two-channel devices were adopted for the Whole Earth Telescope (WET) for its observations of variable white dwarfs. 
For uniformity, each WET participant uses similar equipment, software and observing procedures wherever possible (Nather et al. 1990). Uniformity of observations is one of the WET's most important requirements, permitting it to produce a wealth of new information on its targets (Winget et al. 1990).

For the high-speed work required by the WET observers, however, even the addition of the second channel can leave artifacts in the data. We have found the night sky often varies on similar timescales as do the variable white dwarfs (as quickly as a few minutes). Since the white dwarfs are intrinsically faint, measured pulsation mode amplitudes can therefore be inaccurate without a continuous subtraction of the sky background. Having accurate amplitudes is particularly important in understanding the modes which seem to change their frequencies and power on short timescales. We need to know if the amplitude changes seen are real, or simply consistent with instrumental noise. We therefore realized the need for a three-channel photometer to monitor not only the target and comparison stars, but the sky as well - its purpose, to measure accurate pulsation amplitudes. Other groups had independently come to the same conclusion; see for example Vauclair et al.(1989).

Our first three-channel instrument was a complete re-design and required collaborators to replace their current photometers with the new one in order to get three channels. While this solution was acceptable by our well-funded collaborators with lots of shop time (only one other instrument has been built to date), it was not the solution we needed to outfit a complete network with three-channel instruments. We needed to design a new instrument that would easily work with the existing two-channel photometers, upgrading them to include a third channel with as little modification to the base instrument as possible. We report here on the design of just such an instrument; we call it the dual-beam miniphot. The new instrument has been used in four WET runs and in many supplementary observations as well. We consider the instrument a prototype, and there are a few areas which could be improved. However, the photometer is certainly capable of recording scientifically valuable data and has already demonstrated this. The planned improvements are mainly conveniences and do not significantly affect the quality of the data.

While the primary motivation for the instrument was to obtain more accurate measurements of pulsation amplitudes, observing with three channels has other advantages, many of which we had not anticipated. We have often been able to take useful data with three 
channels when we would have otherwise been forced to close the dome and yield the night to the spectroscopists. In particular, we gain nearly an hour and a half extra observing time each night by starting before and continuing after astronomical twilights. We can extend our monthly observing time, often competing with spectroscopists for grey and bright time, because the sky channel allows an accurate subtraction of varying levels of moonlight. The WET has allowed us to remove the single-day gap that used to occur in our measurements due to the inevitable rising of the Sun; the sky channel allows us to remove the periodic one-month gap that comes from avoiding the Moon.

Because of our increased ability to measure and subtract the sky brightness, we find we can observe dimmer stars (where sky typically dominates the signal) on smaller telescopes than were previously useful. This capability is crucial in extending the WET's eyes to dimmer objects - it is very difficult to get two weeks of overlapping time on many large telescopes around the globe. We have been able to take good data during a variety of adverse weather conditions such as intense northern lights (they do occasionally make it to Texas) and active lightning storms on the horizon.

\section{The instrument}

\section{Criteria of the design}

The criteria for a WET instrument are different from those for a standard single-site instrument. The photometer is often carried as checked-baggage to remote sites and must therefore be relatively light, yet still able to survive the abuse of aggressive baggage handlers. Because we want to equip the entire network with identical instruments, it must also be fairly simple to manufacture and as inexpensive as possible. It could potentially be used on a variety of telescopes, so it must be able to handle a wide range of focal ratios. Since we are talking about an upgrade, it would be nice if it could be easily integrated into the current two-channel photometers, requiring no modifications to the base instrument, and if its use required little change in current operating procedures. The list is a tough one: the instrument needs to be light, rugged, easy to make and operate, flexible and low-cost. 
The scientific requirements of the instrument are equally numerous. It must have a motor-controlled filter wheel with at least 4 available filters to allow for multi-color work. We require a complete set of apertures to accommodate a large variety of seeing conditions and telescope plate scales. To see that the chosen area of sky is not contaminated by nearby stars, there must be an acceptable way of changing the location at which we measure sky. We must also meet the standard photometer requirements that it have illuminated apertures and optics with which to view them, a set of Fabry lenses to provide uniform measurements despite small image drift (Michlovic 1972 gives a simple discussion on this crucial photometer element) and a dark slide to protect the tubes from light. All mating surfaces must be stepped to provide a labyrinth that ensures no light leaks.

There are two additional requirements that we consider so basic that they are usually left unsaid, but as the saying goes, "if it goes without saying, say it twice." The lightpath from the telescope to the ch1 phototube must have nothing in its way other than the Fabry lens. Additional mirrors are often inserted in the lightpath to make the instrument more compact, but at the expense of losing light to the ch1 phototube. Coated mirrors come with $90 \%$ or more reflection guarantees, but add a little dust and a few fingerprints and you can easily lose even $50 \%$ of the light. We are in the business of counting photons; why throw even one away unnecessarily?

The apertures we use also function as a knife-edge for focussing. By removing the eyepiece, and moving the star across the sharp edge of a large aperture, a precise focus can be obtained at the start of each run. If you cannot see the apertures, or they do not have sharp edges, then you lose the ability to accurately focus the telescope to the instrument.

\subsection{The dual-beam miniphot: overall design}

Fig. 1 is a photograph of the Texas two-channel guider box with the dual-beam miniphot attached at the bottom. Fig. 2 is a schematic of the major components of the new instrument only. The miniphot is shown on the offset guider in Fig. 1 and is used for comparison star (Ch2) measurements. For two-channel use, a miniphot is also mounted at the bottom of the instrument for measuring the target star (Ch1). The new three-channel instrument simply replaces the ch1 miniphot with the dual-beam miniphot, adding the sky channel (Ch3) without affecting the rest of the instrument. 


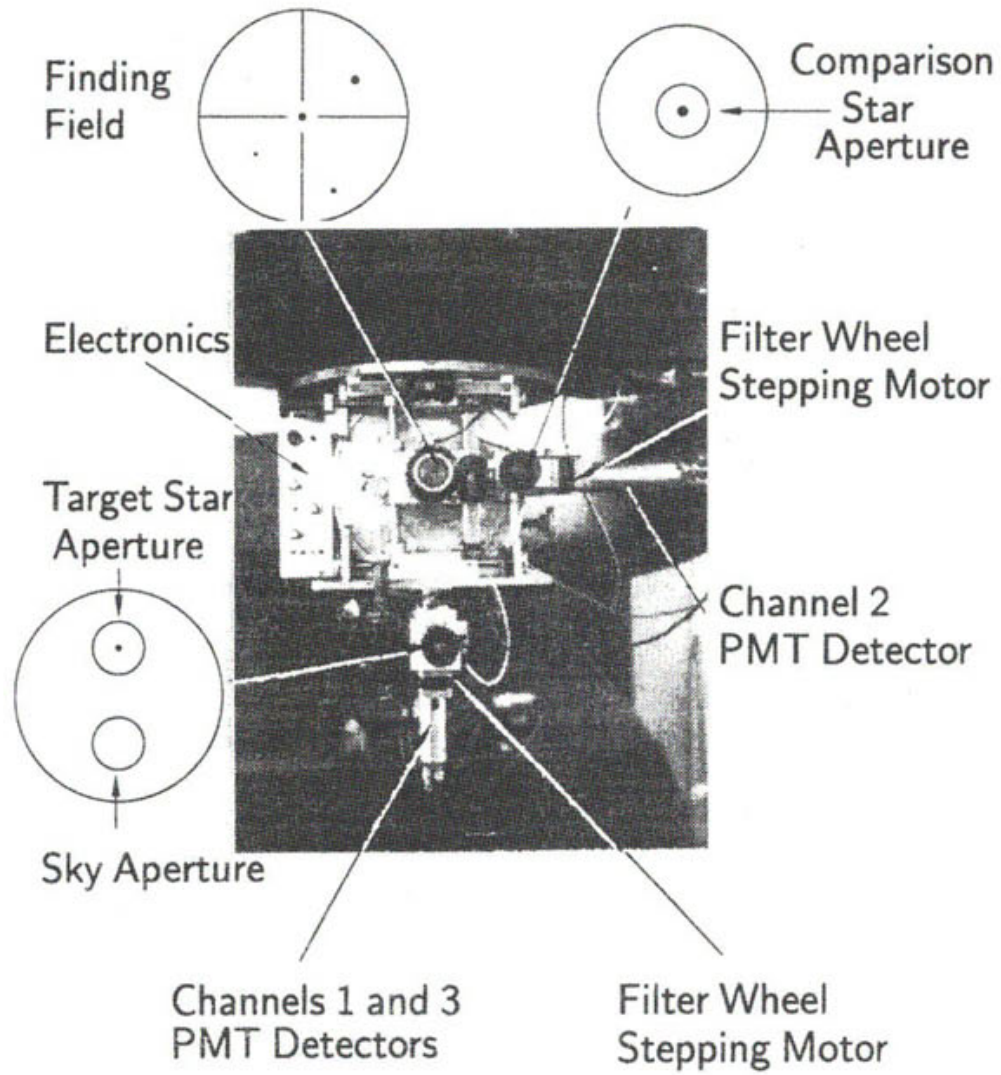

Fig. 1. The WET photometer mounted on the McDonald $36^{\prime \prime}$ telescope and the view from each eyepiece.

The main feature of the dual-beam miniphot design is that two lightbeams are being measured by the instrument at the same time. It has two sets of apertures and filters, two Fabry lenses, and of course two phototubes all housed in a single unit. When looking through the eyepiece, the observer sees a pair of illuminated apertures: one for the target star and one for a clear region of sky. Since the sky aperture is fixed relative to the target aperture, it could be contaminated by a random field star. In all but a few percent of these cases, we can simply swap the star and sky positions. In this way, there are two spots to find an uncontaminated area of sky and a problem only arises when both positions contain a contaminated sky. The dual-beam miniphot therefore has a rotating, detented mount, allowing $360^{\circ}$ of rotation and thus, the ability to select a patch of sky anywhere within a fixed radius of the target star. The detents allow the position to be 


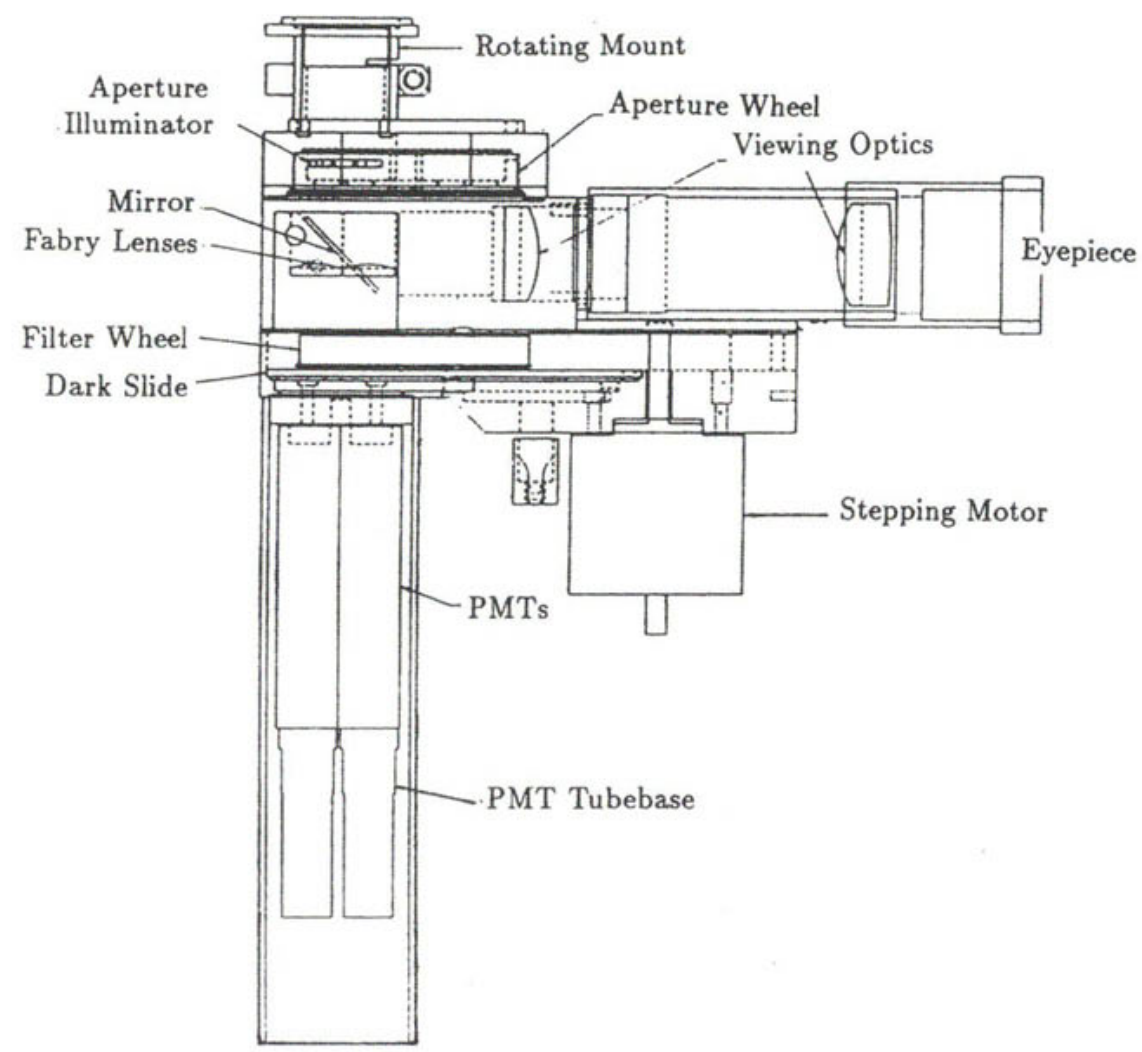

Fig. 2. A layout diagram of the new dual-beam miniphot.

accurately reproduced for further observations, but do not preclude selecting a spot in between detents.

The constraining factor in the design was the size of the available unvignetted field from the guider box (section 2.3 explains this constraint). Because this field is so small, and we want to be able to run on a variety of $F$ ratios (we had aimed for $F / 8$ as our fastest), we could not space the apertures far enough apart to center them over the phototubes. We therefore centered the Fabry lenses over the phototubes and use them to translate the light beam to the phototubes from the apertures (see Fig. 3 to see the schematic lightpath through the system). While this approach is unconventional, certainly, we note no ill-effects by its use. Fabry lenses are traditionally equally spaced between the apertures and the phototubes. While this arrangement provides a uniformly-sized beam, no losses are incurred by changing this placement (except when sending non-parallel light 
through interference filters, which we do not do here). The space constraints in the system did not allow such an equi-distant placement (although it is close to it) of the Fabry lens, but it nonetheless performs quite well without it.

Additionally, Fabry lenses are usually fixed in place so no movement is possible. Due to the same constraints described above, there was simply not enough room in this design to permit this luxury. Instead, the Fabry lenses are in an assembly with the viewing optics pickoff mirror, sliding into place on a heavily detented shaft when data are being taken, and sliding out when the pickoff mirror is in place to view the apertures. With a carefully constructed, loaded detent, the Fabry lenses' position is precisely reproducible. While unconventional, this arrangement works and was the best solution, given the available space.

Where possible, the components of the dual-beam miniphot were designed to be easy (and therefore inexpensive) to build. The McDonald machinists have estimated it actually costs less to machine the dual-beam unit than it does a single-beam miniphot.

\subsection{Design limitations}

As stated earlier, the biggest constraint in the instrument design was trying to fit two independent lightbeams into the unvignetted field made available by the existing guider boxes. It is the size of this field which dictated the separation of the apertures $(8 \mathrm{~mm}$ centerto-center), the resulting length-scale of the instrument, required the Fabry lenses to be slightly off-axis (see Fig. 3), and limits the workable $F$ ratios. The observer can use the largest apertures $(4 \mathrm{~mm})$ at $F$ ratios up to $F / 10.1$, but is limited to smaller apertures at faster $F$ ratios. At $F / 9$ all but the largest aperture are usable. The internal lightpath makes anything faster than about $F / 8$ unusable.

It would be possible to avoid both the need to bend the lightpath and the limitations at fast $F$ ratios by increasing the unvignetted field size from the guider box. The limiting element determining the field size is the central hole of the large $45^{\circ}$ mirror in the guider box. Were we to relax our criteria and allow modifications to the existing equipment, we could slightly enlarge this hole and increase our available field at the expense of comparison star search area, although the loss here would be unimportant. With such a modification, the apertures in the dual-beam miniphot could be further separated, keeping the entire lightpath in the instrument on-axis and allowing enough 


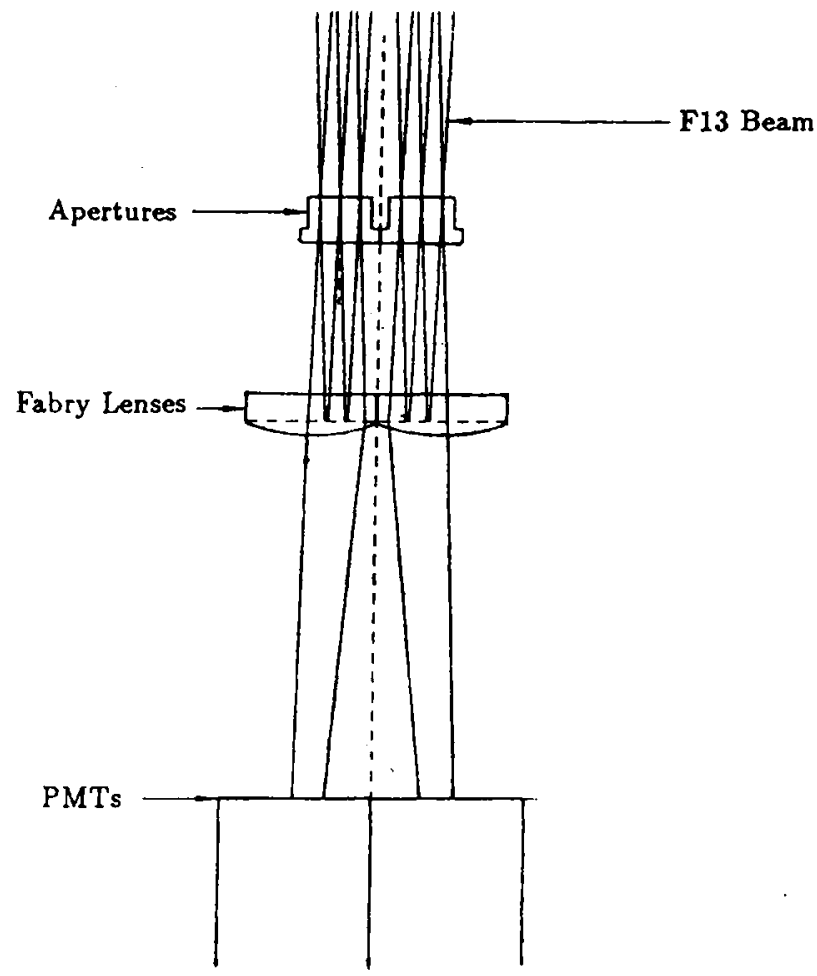

Fig. 3. The lightpath through the dual-beam miniphot.

room to operate at smaller $F$ ratios. This is a possible modification for the next generation instrument, although an increased aperture separation is not without problems of its own - such as fitting both apertures comfortably into the field of view of the aperture eyepiece with enough magnification to make centering a star in the smallest aperture possible.

One last piece that could be redesigned in a future version of the instrument is the rotating mount. Its combination of round and square features make machining it very difficult, which while not ideal, would be acceptable were its performance completely satisfactory. It is not. The current detents are not strong enough to be easily felt and the mount itself is prone to flexure if a force (like an abnormally tight cable) pulls on it. It is capable of rotation, and under normal conditions does not flex, but its overall performance does not match the difficulty of machining it. A likely alternative will be a simpler system based on a circular bearing and a stronger detent. 


\section{Results}

Our primary goal in building the dual-beam miniphot was to increase our ability to measure pulsation amplitudes. Having used the earlier three-channel photometer, we knew this goal would be easily reached. To demonstrate this effect, we used the data set from the May 1994 WET run on the DB white dwarf variable, GD 358, where we had a pair of these new WET standard photometers in operation: one at the $60 \mathrm{~cm}$ telescope at the Siding Spring Observatory in Australia, and one used on the $90 \mathrm{~cm}$ and $208 \mathrm{~cm}$ telescopes at the McDonald Observatory. Selecting only runs long enough to resolve neighboring pulsation modes, we took one fairly isolated mode and measured its amplitude in each run. Fig. 4 has the WET photometer data plotted (and offset by $40 \mathrm{mma}$ for clarity) and all other data (which include some other 3-channel instruments). Most of the telescopes in the network are $1-2 \mathrm{~m}$ in diameter. We determined the mode's amplitude by a linear least-squares fit to each light curve of a sine curve with a fixed period, taken from the overall Fourier transform of the data set. The formal uncertainties on each point are roughly equal and usually of the same order as the size of the plotted symbols. The results show the data from the new instruments to have a mean of $(13.9 \pm 6) \mathrm{mma}$ compared to $(17.7 \pm 9)$ mma for the rest of the data set. The new instruments produced measured pulsation amplitudes $50 \%$ more accurate than the other WET photometers.

Note, however, the scatter in Fig. 4 is not entirely random; indeed it appears to be periodic with a period of roughly four days, likely caused by the beating of two closely-spaced frequencies. The mode we chose to measure is actually one member of a multiplet of closely-spaced modes with an intra-mode spacing of about $6 \mu \mathrm{Hz}$, corresponding to a beat period of almost two days, close to the observed variation. A hint of this variation is also seen in the upper panel of Fig. 4, but it is quite obvious in the WET photometer data. At any rate, the scatter is not random and is likely to be intrinsic to the star, making the WET photometer amplitudes even more precise than the standard deviation would suggest.

We have often claimed that with two channels on our photometer, we could correct for thin clouds simply by dividing the Ch1 light curve by Ch2. In practice, however, it never really worked well. Whereas transparency variations, such as clouds and extinction, affect both light curves the same percentage, sky adds directly to the light measured and depending on the brightness of the observed stars, 


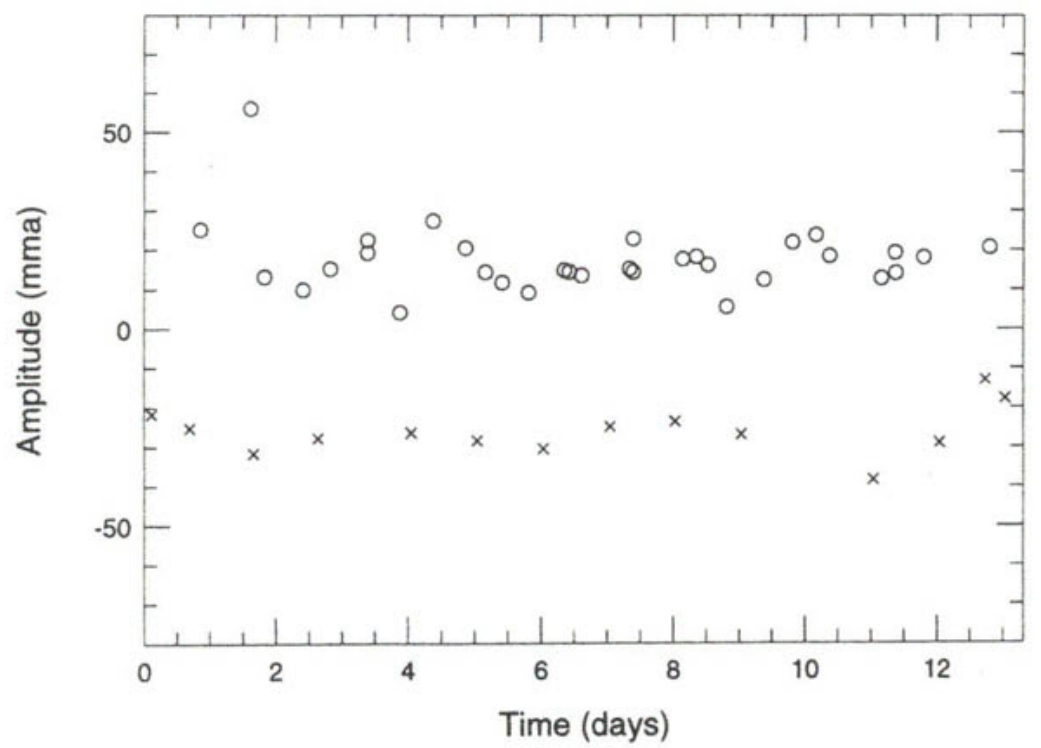

Fig. 4. Amplitudes of a pulsation mode measured in a WET run by standard WET photometers $(x$ - displaced by $40 \mathrm{mma})$ and all others in the network $(0)$.

is a different percentage of the actual number of photons counted. In general then, dividing two light curves with unequal sky-to-star ratios is ineffective. The sky background can be considered a DC offset and therefore division does not completely remove it. The results are particularly bad with moonlit clouds if the stars are of unequal brightnesses. With the sky channel accurately removing the sky background from the data, however, some cloud correction is now possible.

Fig. 5 is a run taken with the new WET photometer on the $60 \mathrm{~cm}$ telescope at the Siding Spring Observatory, Australia. The run was started about $20 \mathrm{~min}$ before evening twilight and continued roughly 30 min past morning twilight. The top panel is the unreduced light curve from the target star, PG 1159-035, showing both the effects of twilight and of occasional passing clouds. The middle panel is the same light curve, just with the sky subtracted. The bottom panel is a semi-reduced light curve, with the sky subtracted, atmospheric extinction corrected and our cloud correction routines applied. The $y$-scale of each plot is different to accommodate the true range in the data. The onset of twilight is now undetectable and some of the 
clouds have been effectively removed. Note that where the cloud is not completely corrected, the original counts had fallen nearly $85 \%$; we can't correct for that much cloud extinction. The increase in noise at each end of the light curve is due primarily to the heavy atmospheric extinction correction needed, and partly to the noise that accompanies the large sky counts during twilight. In practice, we would not use all these data, but the figure shows that some corrections can be made with the third channel that are not possible without it.

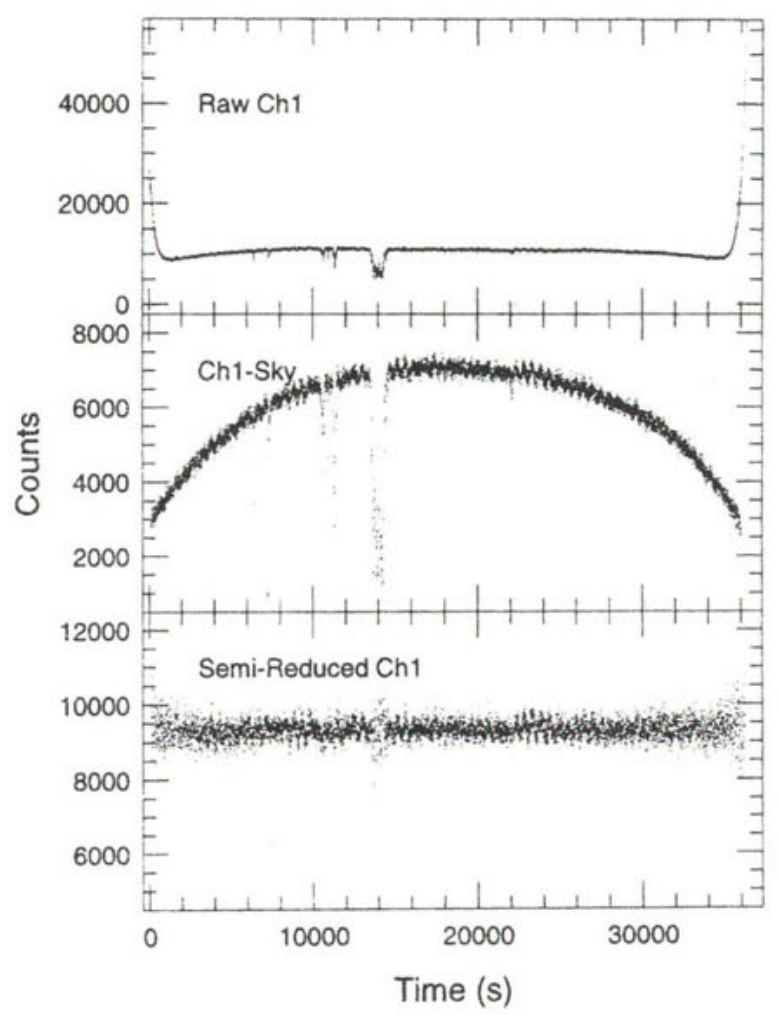

Fig. 5. Observing in twilight and cloud with the WET photometer.

Two more figures show some of the conditions under which we have taken data with the new instrument we couldn't have otherwise. Both Figs. 6 and 7 are from runs taken with three-channel instruments on the $90 \mathrm{~cm}$ telescope at the McDonald Observatory in Texas. The target object in Fig. 6 is PG 1351+489, a low-amplitude, 
$B \approx 16 \mathrm{mag}, \mathrm{DB}$ pulsator. The left-half of the top three panels of the figure are the raw data for each channel: PG 1351, comparison star and sky. Note that it is nearly impossible to spot which curve is the real variable. The bottom two panels show the reduced $\mathrm{Ch} 1$ and Ch2 data (with the sky subtracted), leaving a nearly flat comparison star and an obvious variable in ch1. To the right of each light curve is a Fourier transform (FT) of the data. In the first and second panels, the data are reduced normally, except a constant equal to the mean value of sky is subtracted from the data, instead of the continuous sky subtraction performed in the last two panels. Since the mean sky value is a factor of three times the mean Ch1 counts and one third the $\mathrm{Ch} 2$ counts, the size of the noise introduced in the FT differs in each panel; for Ch1, the noise goes off scale to nearly 170 mma. The reduced Ch1 FT clearly shows the expected periodicity near $2000 \mu \mathrm{Hz}$ (500 s) which is also present in the uncorrected FT, but almost lost in the noise. A peak half this amplitude would be completely lost in the noise: present, but not detected.

The data shown in Fig. 7 are of the DA pulsator G 29-38, taken in clear skies (starting shortly before twilight), but surrounded by lightning. The top panel is the raw target data, while the lower panel shows the sky-subtracted data. Without the sky channel, most of the run would simply be too noisy to be of much scientific value. As in the case of the data in Fig. 6, an occasional measurement of the sky taken every 20 min would leave the artifacts in the data. The lightning, however, is nicely removed from the data by subtracting the continuous sky channel. The same is with other cases of scattered light, like moonlight reflecting off a rotating dome, car headlights and stray flashlight beams.

\section{Conclusion}

The Whole Earth Telescope has made remarkable instrumental progress that has made the asteroseismological studies of white dwarfs possible. One further instrumental step was required that would allow the WET to obtain more accurate pulsation amplitude information, extend available observing time to include grey and bright time and allow useful data to be obtained on dimmer stars with smaller telescopes. Adding a sky channel to the existing twochannel photometers meets all the above objectives. We therefore designed a new dual-beam miniature photometer that is a bolt-on upgrade to turn existing two-channel photometers into three-channel 


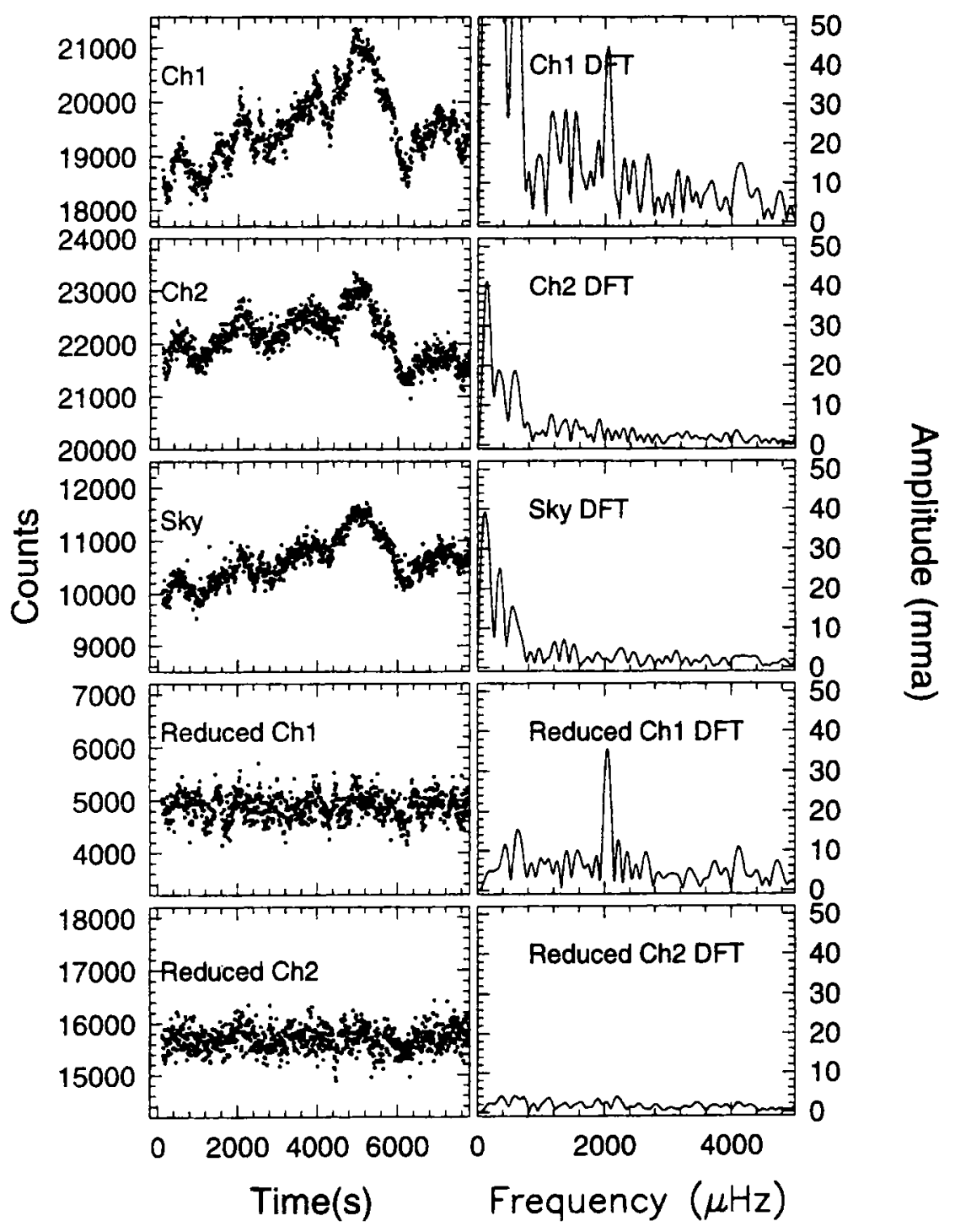

Fig. 6. Three channel data taken during extreme sky variations.

photometers that have the new ability to measure the sky brightness continuously. We will make the plans of the new instrument (including the electronics, computer interface and software) available to all interested parties on request. 


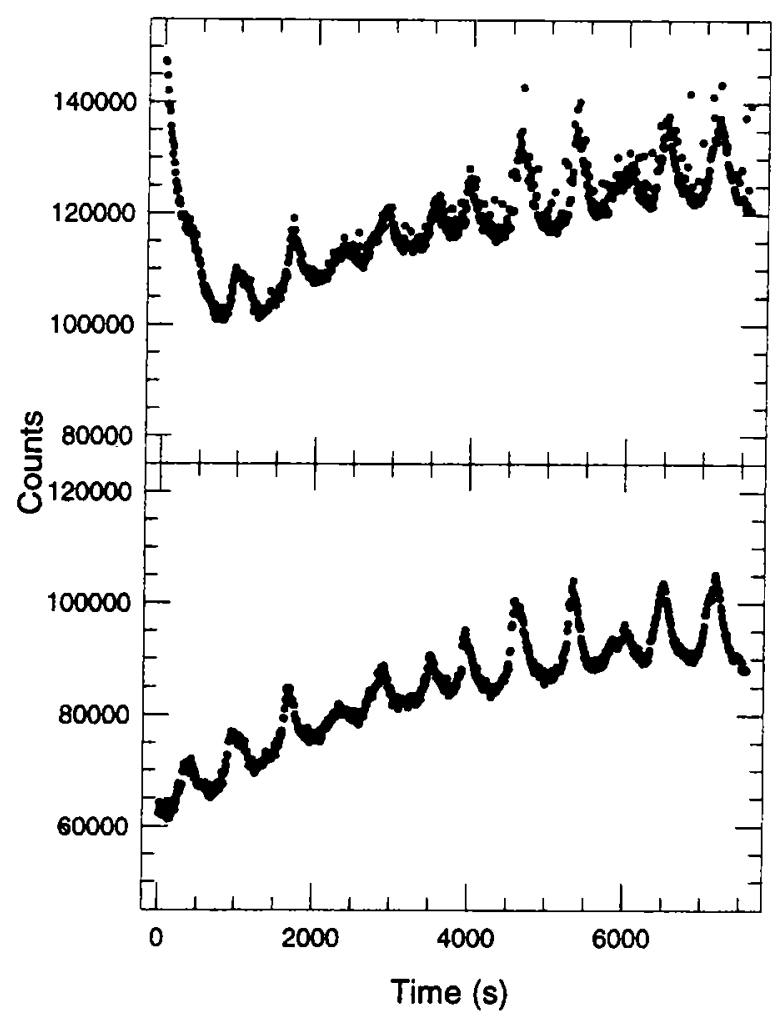

Fig. 7. Three channel data taken with surrounding lightning.

To summarize, we present here the principal characteristic of the WET photometer and those that any other photometer must also have to be considered an acceptable replacement:

- three counting channels: target star, comparison star and sky;

- no reflection optics in channel 1 (coated Fabry lens only);

- $\mu$-metal shielded bi-alkali PMT detectors in all channels (Hamamatsu R647);

- adjustable apertures in all channels, concentric, identical in size;

- independent filters in all channels, after Fabry lens, motordriven;

- provision for guiding without interrupting the data channels;

- precise telescope focussing method (e.g. knife-edge);

- unitized TTL pulse output from PMT preamplifier/discriminator;

- live, real-time graphical display of the 3 incoming light curves;

- portability. 


\section{References}

Grauer A.D., Bond H.E. 1981, ApJ, 93, 388

Michlovic J. 1972, Applied Optics, 11, 490

Nather R.E. 1973, Vistas in Astronomy, 15, 91

Nather R.E., Winget D.E., Clemens J.C., Hansen C.J., Hine B.P. 1990, ApJ, 361, 309

Vauclair G., Goupil M.J., Baglin A., Auvergne M., Chevreton M. 1989, A\&A, 215, L17

Winget D.E. et al. 1990, ApJ, 357, 630 